\title{
Biological signatures of brain damage associated with high serum ferritin levels in patients with acute ischemic stroke and thrombolytic treatment
}

\author{
Mónica Millán $^{\mathrm{a}, *}$, Tomás Sobrino ${ }^{\mathrm{b}}$, Juan Francisco Arenillas ${ }^{\mathrm{a}}$, Manuel Rodríguez-Yáñez ${ }^{\mathrm{b}}$, \\ María García ${ }^{\mathrm{c}}$, Florentino Nombela ${ }^{\mathrm{d}}$, Mar Castellanos ${ }^{\mathrm{e}}$, Natalia Pérez de la Ossa ${ }^{\mathrm{a}}$, Patricia Cuadras ${ }^{\mathrm{f}}$, \\ Joaquín Serena ${ }^{\mathrm{e}}$, José Castillo ${ }^{\mathrm{b}}$ and Antoni Dávalos ${ }^{\mathrm{a}}$ \\ ${ }^{a}$ Department of Neurosciences, Hospital Germans Trias i Pujol, Departament de Medicina, Universitat Autònoma \\ de Barcelona, Spain \\ ${ }^{\mathrm{b}}$ Department of Neurology, Clinical Neuroscience Research Laboratory, Hospital Clínico Universitario, \\ Universidad de Santiago de Compostela, Spain \\ ${ }^{\mathrm{c}}$ Unit of Bioestatistics, Hospital Doctor Josep Trueta, Girona, Spain \\ ${ }^{\mathrm{d}}$ Department of Neurology, Hospital de la Princesa, Madrid, Spain

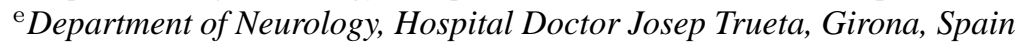 \\ ${ }^{\mathrm{f}}$ Department of Radiology, Hospital Germans Trias i Pujol, Departament de Medicina, Universitat Autònoma de \\ Barcelona, Spain
}

\begin{abstract}
Background and purpose: Increased body iron stores have been related to greater oxidative stress and brain injury in clinical and experimental cerebral ischemia and reperfusion. We aimed to investigate the biological signatures of excitotoxicity, inflammation and blood brain barrier disruption potentially associated with high serum ferritin levels-related damage in acute stroke patients treated with i.v. t-PA.

Methods: Serum levels of ferritin (as index of increased cellular iron stores), glutamate, interleukin-6, matrix metalloproteinase-9 and cellular fibronectin were determined in 134 patients treated with i.v. t-PA within 3 hours from stroke onset in blood samples obtained before t-PA treatment, at 24 and 72 hours.

Results: Serum ferritin levels before t-PA infusion correlated to glutamate $(r=0.59, p<0.001)$ and interleukin-6 $(r=0.55$, $p<0.001)$ levels at baseline, and with glutamate $(r=0.57, p<0.001)$, interleukin-6 $(r=0.49, p<0.001)$, metalloproteinase-9 $(r=0.23, p=0.007)$ and cellular fibronectin $(r=0.27, p=0.002)$ levels measured at 24 hours and glutamate $(r=0.415$, $p<0.001)$, interleukin-6 $(r=0.359, p<0.001)$ and metalloproteinase-9 $(r=0.261, p=0.004)$ at 72 hours. The association between ferritin and glutamate levels remained after adjustment for confounding factors in generalized linear models.

Conclusions: Brain damage associated with increased iron stores in acute ischemic stroke patients treated with iv. tPA may be mediated by mechanisms linked to excitotoxic damage. The role of inflammation, blood brain barrier disruption and oxidative stress in this condition needs further research.
\end{abstract}

Keywords: Iron stores, thrombolysis, ferritin, biomarkers, excitotoxicity, blood-brain-barrier disruption, inflammation

\footnotetext{
*Corresponding author: Mónica Millán, MD, Stroke Unit. Department of Neurosciences, Hospital Germans Trias i Pujol, Departament de Medicina, Universitat Autònoma de Barcelona, 08916
}

Badalona, Barcelona, Spain. Fax: +34 93497 8742; E-mail: mmillan.germanstrias@gencat.net. 


\section{Background}

The knowledge of new factors associated with a poor response to thrombolysis in stroke patients may help to conceive new strategies to improve the benefit of this treatment. It has recently been shown that high serum ferritin levels are associated with poor functional outcome, hemorrhagic transformation and severe brain edema in patients treated with i.v. tissue plasminogen activator (t-PA) after ischemic stroke. These findings indicate that increased body iron stores may offset the beneficial effect of thrombolytic therapy [19].

The release of free iron from intracellular stores such as ferritin as a result of cerebral ischemia, particularly during reperfusion, catalyses the generation of the toxic free radical hydroxyl which destroys cellular and microvascular integrity [16,27]. Experimental data supports the key role played by iron in brain and endothelial injury in cerebral ischemia and reperfusion. Iron intake has been associated with larger infarct volume, greater reactive oxygen species (ROS) generation in brain and peripheral vasculature, glutamate release and inflammatory response after middle cerebral artery occlusion in the rat $[2,18]$, whereas iron chelators, antioxidants or ROS scavengers have shown neuroprotective effects reducing infarct size, brain edema, ROS production and metabolic failure in ischemia/reperfusion experimental stroke models $[9,11,24,26]$. In addition, cellular biological studies in animal models have found a close link between ROS generation in microvessels and astrocytic end-feet, increased matrix metalloproteinases9 (MMP-9) expression and blood brain barrier (BBB) disruption [12].

Taking together, these findings suggest that generation of ROS during reperfusion, increased excitotoxic damage, inflammation and BBB disruption might be potential mechanisms of greater brain and endothelial injury in ischemic stroke patients with iron overload who are treated with i.v. t-PA. However, the underlying pathophysiological basis of brain damage in these patients is unknown. The aim of this study was to investigate the biological signatures potentially associated with high serum ferritin levels-related damage in acute stroke patients treated with i.v. t-PA by measuring a series of biomarkers of excitotoxicity, inflammation and endothelial injury that have been validated in previous clinical studies.

\section{Patients and methods}

We studied acute ischemic stroke patients treated with i.v. t-PA within 3 hours from symptom onset in four university hospitals who accomplished the SITSMOST criteria [32]. Patients were continuously monitored during the first 24 hours in the acute stroke unit and were prospectively evaluated using brain CT, and neurological and functional scales according to the SITS-MOST registry during a follow-up period of 90 days. The protocol was approved by the Ethics Committees of the participating centers and informed consent was signed by patients or their relatives. For the purpose of this investigation, additional exclusion criteria were prior disability (modified Rankin Scale (mRS) $>1$ ), alcohol consumption $\geqslant 40 \mathrm{~g} /$ day, current treatment with iron, and known infectious, inflammatory or cancer diseases at the time of treatment.

\subsection{Clinical variables}

Stroke severity was quantified before t-PA administration and at 24 hours by using the National Institute of Health Stroke Scale (NIHSS). Early neurological deterioration was diagnosed when the NIHSS worsened $\geqslant$ 4 points between baseline and $24 \mathrm{~h}$. Poor outcome was defined as mRS score $>2$ at 90 days.

\subsection{Neuroimaging variables}

CT scans were carried out on admission, and at 2436 hours after thrombolytic therapy. Early CT signs of infarction were evaluated on admission, and infarct volume, hemorrhagic transformation (HT) and brain edema were assessed at 24-36 hours. HT was classified as hemorrhagic infarction, and parenchymal hematoma (PH) according to the ECASS-2 criteria [17]. PH was considered as being symptomatic when it was associated with early neurological deterioration. Severe brain edema was diagnosed if there was extensive swelling causing any shifting of the midline structures. Hypodensity volume was calculated using the formula $0.5 \mathrm{x}$ $\mathrm{a} \times \mathrm{b} \times \mathrm{c}$, where $\mathrm{a}$ and $\mathrm{b}$ are the largest perpendicular diameters, and $\mathrm{c}$ is the slice thickness. CT scans were evaluated by investigators blinded to the laboratory determinations and clinical outcome.

\subsection{Laboratory determinations}

Serum samples were taken immediately after admission (within 3 hours of stroke onset and before the t-PA treatment), at 24 hours and at 72 hours after the t-PA bolus infusion, and they were stored at $-80^{\circ} \mathrm{C}$. Serum ferritin levels, as an index of body iron stores, were determined by electrochemiluminescence immunoassay 
using an analyzer ELECSYS 2010 (Roche Diagnostics $\mathrm{GmbH})$; glutamate levels, as a biomarker of excitotoxicity, were determined by HPLC, using the Waters Pico Tag ${ }^{\circledR}$ Chemistry Package for HPLC amino acids analysis; interleukin-6 (IL-6), as an index of inflammatory response, were determined by IMMULITE 1000 System, Diagnostic Products Corporation (DPC), USA; and finally, c-Fn and MMP-9 levels, as BBB disruption biomarkers, were determined with commercially available quantitative sandwich enzyme-linked immunoabsorbent assay kits obtained from Biohit Plc, Finland, and Biotrack, Amersham Pharmacia, UK, respectively. Biomarker concentrations were measured in a central laboratory by investigators blind to the clinical outcome and neuroimaging findings. Clinical investigators were unaware of the laboratory results until the end of the study, once the data base was closed.

\subsection{Statistical analyses}

Categorical variables were expressed as proportions and compared by using the $\chi^{2}$ test. Continuous variables were expressed as the mean and SD when the variable distribution was normal or median and quartiles when it was not normal, and compared by the Student's t-test or the Mann-Withney test, respectively. Spearman's coefficient was used to analyze the correlation between serum ferritin levels, biomarker concentrations and other continuous variables. Receiver operating characteristic (ROC) curves were configured to establish cut-off points of ferritin level that optimally predicted the occurrence of poor outcome. Accordingly, the impact of serum ferritin levels on outcome, HT and brain edema formation was assessed by logistic regression analysis adjusting for age, stroke severity and other baseline variables related in the univariate analysis $(p$ value $<0.1$ ). To assess the influence of baseline ferritin levels on the serum concentrations of brain injury biomarkers, general linear models for repeated measurements were used and they were adjusted for variables related to baseline ferritin concentrations with a $p$ value $<0.05$.

\section{Results}

A total of 134 patients treated with t-PA were included in this study. Reasons for exclusion and baseline characteristics of included patients have been recently reported [19]. Blood samples were obtained in all patients at baseline, in 130 patients at 24 hours (one patient died before, and samples from 3 patients were unavailable) and in 118 patients at 72 hours ( 3 patients died, 6 were discharged before the third day, and samples from 9 patients were unavailable). No patients were lost of follow-up.

\subsection{Clinical outcome, neuroimaging findings and biomarkers of brain injury}

At 3 months, poor outcome was recorded in 73 patients $(54.5 \%)$, and mortality in 18 patients $(13.4 \%)$. CT at 24-36 hours showed no signs of infarction in $10 \%$ of patients. Median hypodensity volume was 19 cc $[3,80]$. HT was observed in $27(20 \%)$ patients, hemorrhagic infarction in 15 and $\mathrm{PH}$ in 12 patients (symptomatic in 4), and severe cerebral edema was found in $15(11.2 \%)$ patients.

Table 1 shows the baseline characteristics, laboratory parameters, and brain injury biomarkers in subgroups classified by clinical outcome at 90 days, presence of PH, and severe brain edema at 24-36 hours after treatment. Baseline serum ferritin, glutamate, IL-6, c-Fn and MMP-9 levels were significantly higher in patients with poor outcome, $\mathrm{PH}$ and severe brain edema in univariate analyses (Table 1).

As previously reported the adjusted odds ratios of poor outcome, $\mathrm{PH}$ and severe brain edema for ferritin levels $>79 \mathrm{ng} / \mathrm{mL}$ were 117 [95\%CI, 24.4-559.5], 4.2 [95\%CI, 0.8-22], and 6.4 [95\%CI, 1.3-32], respectively [19].

\subsection{Serum ferritin and biomarkers of brain injury}

Serum ferritin profile during the first 72 hours showed a parallel slight increase in both outcome groups (data showed in ref. 19). Baseline serum ferritin concentrations were significantly higher in patients with diabetes mellitus ( $p=0.041)$, early CT signs of infarction $(p<0.001)$, and in non-lacunar stroke subtype ( $p=0.005$ ), but no differences were found for sex and other vascular risk factors. Baseline ferritin values correlated with NIHSS at admission $(r=0.489, p<$ $0.001)$ and at 24 hours $(r=0.747, p<0.001)$, and with infarct volume $(r=0.663, p<0.001)$. No correlations were found with age, systolic and diastolic blood pressure, body temperature, serum glucose, platelet count, leukocytes, aPTT and time from symptoms onset to t-PA bolus.

Table 2 shows the correlations between ferritin levels at baseline and the biomarkers of brain injury measured at different intervals. A significant correlation was 


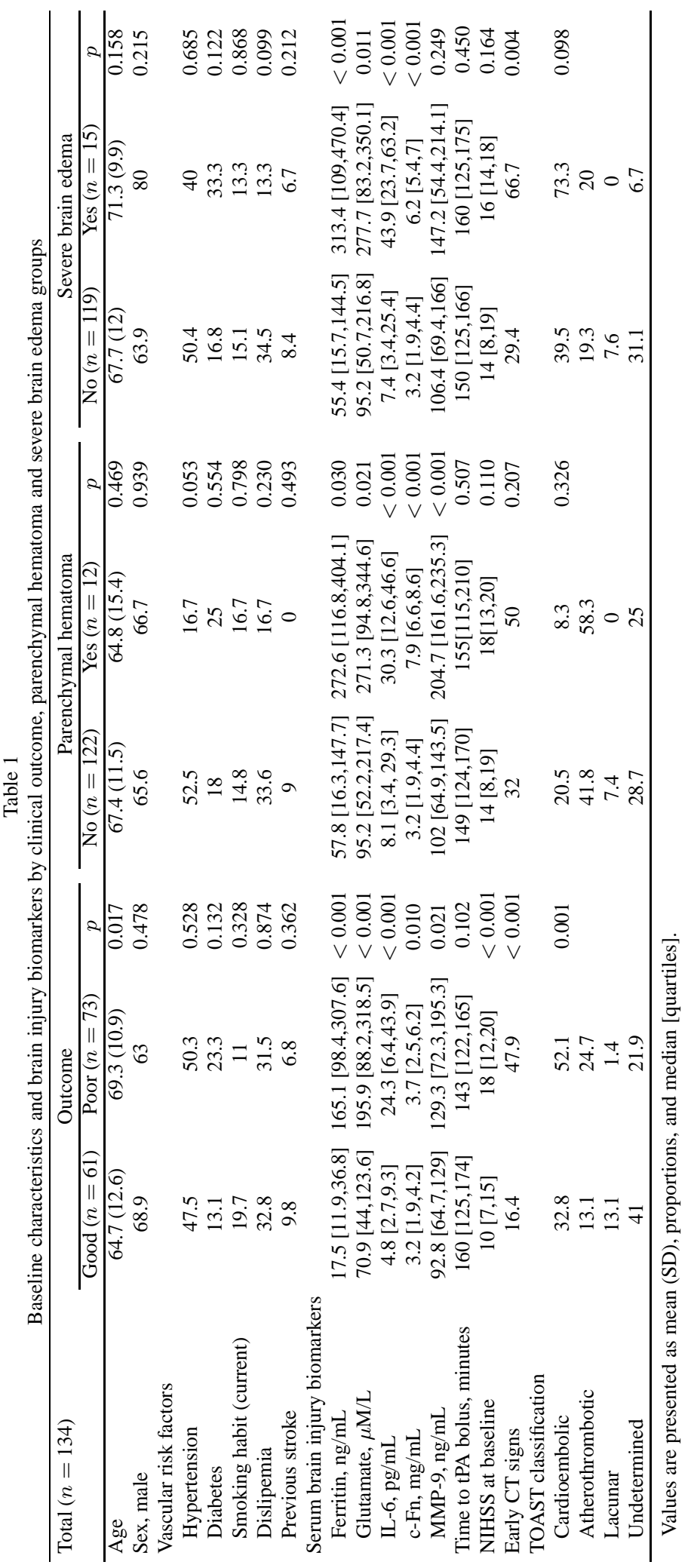


Table 2

Correlation coefficients between baseline ferritin levels and the biomarkers of brain injury

\begin{tabular}{lccc}
\hline Biomarkers & Baseline & 24 hours & 72 hours \\
\hline Glutamate, $\mu \mathrm{M} / \mathrm{L}$ & $r=0.589, p<0.001$ & $r=0.507, p<0.001$ & $r=0.415, p<0.001$ \\
IL-6, pg/mL & $r=0.549, p<0.001$ & $r=0.465, p<0.001$ & $r=0.359, p<0.001$ \\
c-Fn, mg/L & $r=0.154, p=0.075$ & $r=0.268, p=0.002$ & $r=0.096, p=0.301$ \\
MMP-9, ng/mL & $r=0.114, p=0.190$ & $r=0.234, p=0.007$ & $r=0.261, p=0.004$ \\
\hline
\end{tabular}

Table 3

Adjusted baseline ferritin concentrations for serum biomarker levels at repeated measurements

\begin{tabular}{lcc}
\hline Models & $\beta(95 \% \mathrm{CI})$ & $p$ \\
\hline Model 1 & \\
Serum glutamate, $\mu \mathrm{M} / \mathrm{L}$ & $0.42(0.25,0.59)$ & $<0.001$ \\
At baseline & $0.26(0.11,0.42)$ & 0.001 \\
At 24 hours & $0.16(0.02,0.30)$ & 0.020 \\
At 72 hours & & \\
Model 2 & $0.014(-0.011,0.03 .9)$ & 0.233 \\
Serum IL-6, pg/mL & $0.014(-0.032,0.060)$ & 0.541 \\
At baseline & $-0.007(-0.080,0.068)$ & 0.861 \\
At 24 hours & & \\
At 72 hours & $0.001(-0.003,0.004)$ & 0.640 \\
Model 3 & $0.003(-0.001,0.006)$ & 0.148 \\
Serum c-Fn, mg/L & $0.001(-0.003,0.004)$ & 0.791 \\
At baseline & & \\
At 24 hours & & \\
At 72 hours & & 0.521 \\
Model 4 & $0.036(-0.140,0.074)$ & 0.710 \\
Serum MMP-9, ng/mL & $0.018(-0.078,0.110)$ & 0.013 \\
At baseline & $0.130(0.028,0.230)$ & \\
At 24 hours & At 72 hours &
\end{tabular}

The models were adjusted for history of diabetes mellitus, early CT signs, stroke severity and non-lacunar stroke subtype.

found between baseline ferritin levels and the concentrations of glutamate and IL-6 on admission, at 24 and at 72 hours. Baseline serum ferritin levels did not correlate with either c-Fn or MMP-9 on admission, whereas a slight correlation was found between baseline ferritin and c-Fn and MMP-9 levels at 24 hours (Fig. 1), and between baseline ferritin and MMP-9 levels at 72 hours.

The association between ferritin and glutamate levels remained significant after adjustment for diabetes mellitus, early CT signs, stroke severity, and non-lacunar stroke subtype in generalized linear models for repeated measurements. However, IL-6, cFN and MMP-9 levels at repeated samples lost their association with baseline ferritin after adjustment for covariates (Table 3).

\section{Discussion}

High serum ferritin concentrations, used as an index of increased body iron stores, have been previously reported to be associated with poor outcome, parenchymal hematoma and severe brain edema in this same sample of patients [19]. In this secondary study, we have investigated the biological signatures of brain damage associated with increased body iron stores by analyzing a series of accepted biomarkers of brain injury. Baseline ferritin levels showed a moderate correlation with glutamate and IL-6 and a slight correlation with the biomarkers of BBB injury. Importantly, the association with high glutamate levels over the first 72 hours after admission remained independent of other related clinical variables. These findings support that excitotoxic damage might mediate the greater brain injury found in ischemic stroke patients with increased ferritin levels that are treated with t-PA.

The tested biomarkers have widely been assessed in previous studies and validated as early and good biological predictors of brain and BBB injury $[3,4,6,7$, $21,28,29,31]$. Furthermore, we have recently demonstrated that high levels of glutamate in the peripheral blood are associated with ischemic lesion growth on diffusion-weighted MRI in the acute phase of ischemic stroke [5]. In fact, the present study confirms that elevated serum concentrations of glutamate, IL-6, cFn and MMP-9 on admission are associated with poor functional outcome, $\mathrm{PH}$ and severe brain edema in patients with ischemic stroke treated with iv. t-PA. The association between high ferritin levels and glutamate release in serum and cerebrospinal fluid was also found in a series of patients with acute stroke not treated with thrombolysis [10]. Enhanced glutamate-mediated excitotoxicity was proposed as one of the mechanism of increased body iron stores related injury in cerebral ischemia since, in neuronal cultures, glutamate release is significantly increased by systems leading to free radical formation [22,25], whereas antioxidants have shown a neuroprotective effect by decreasing glutamate release and subsequent excitotoxicity in experimental stroke models [14].

The present findings are in line with experimental data in a rat model of permanent MCA occlusion after high iron intake which showed $66 \%$ greater infarct volume, and higher serum levels of glutamate, IL-6, tumour necrosis factor- $\alpha$ and thiobarbituric acid reaction substances (TBARS) in comparison with those of ani- 
A)
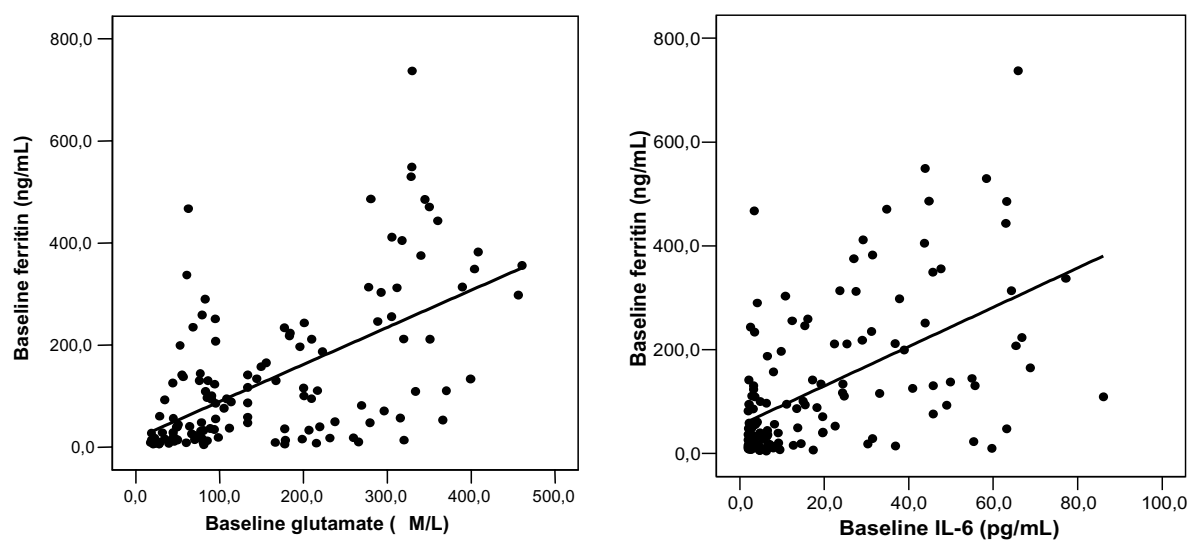

B)
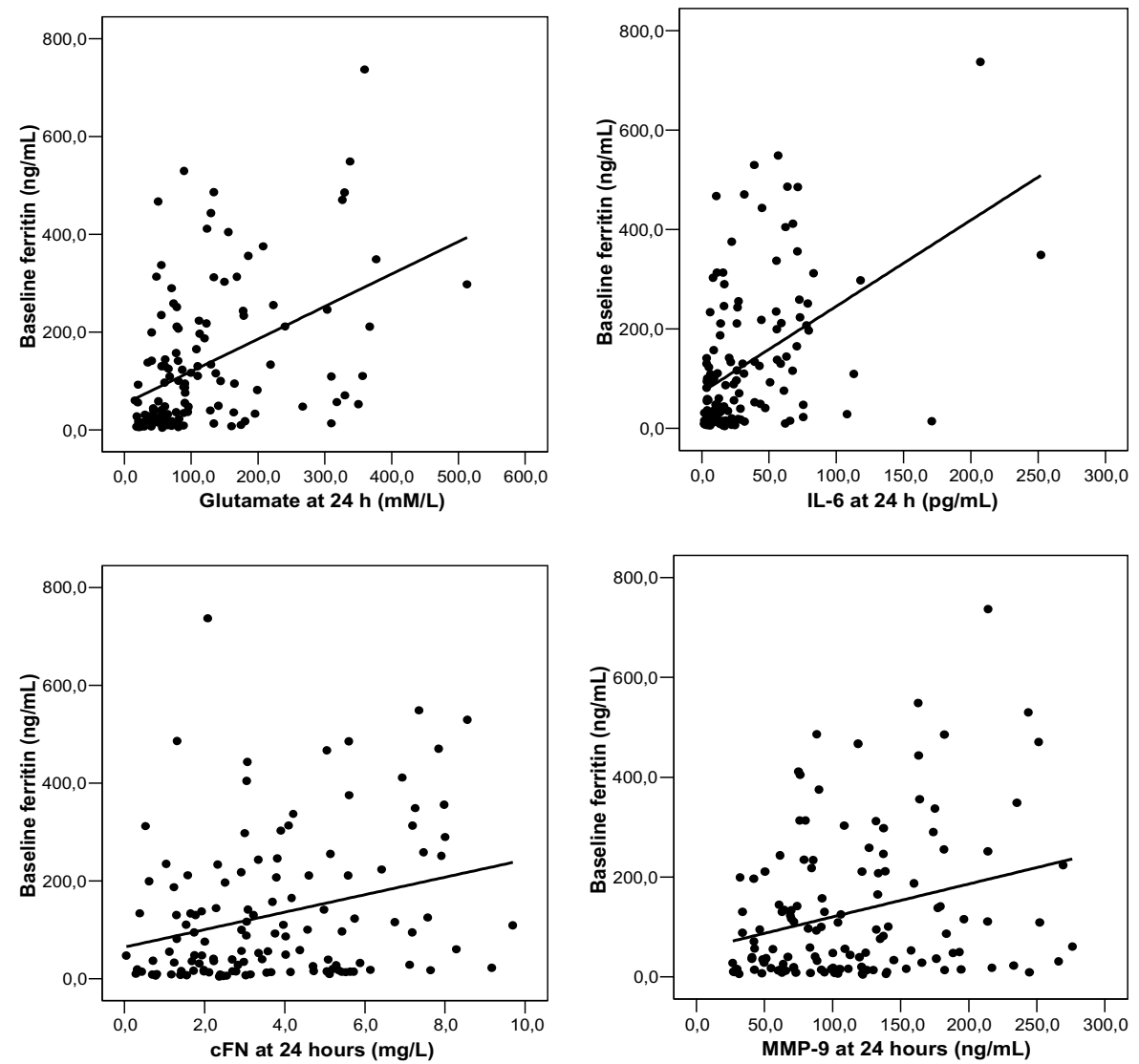

Fig. 1. Significant correlations between serum ferritin and serum biomarkers concentrations at baseline (A) and at 24 hours (B). Correlation coefficients are shown in Table 2.

mals receiving a normal diet [2]. Therefore, biomarkers of oxidative stress, excitotoxicity and inflammation could be surrogates of the importance of iron-related brain damage after cerebral ischemia. These biomarkers are likely reflecting distinct mechanisms of injury which are interconnected, so it is difficult to delineate 
the role of each of them.

The deleterious effects of iron in the neurovascular unit might be aggravated by the t-PA-induced excitotoxicity and MMP-9 overexpression [15,23,30], and by a higher free radicals generation from oxygen after tPA-induced reperfusion [1], leading to HT and edema. In fact, although iron overload in animals has not been associated with changes in cerebral total iron levels [2], iron accumulates in the endothelial cells [18] and, in ischemia-reperfusion stroke models, it is significantly increased in some brain areas such as the hippocampus along with BBB disruption, lipid peroxidation and apoptosis [13]. The lack of an independent association of serum ferritin values with the markers of inflammatory response and $\mathrm{BBB}$ disruption is not in favor of an enhancing effect of iron overload on the mechanisms underlying the microvascular disruption after cerebral ischemia in patients treated with trhombolytic drugs. However this hypothesis needs to be tested in experimental studies.

This study has a number of limitations. First, serum biomarkers do not necessarily reflect the importance of the molecular processes in the ischemic brain, and may be influenced by the acute phase reaction associated with cerebral ischemia. However, serial determinations have not shown a significant increase of serum ferritin levels $4 \mathrm{~h}$ after experimental stroke, and inconsistent results at 24 hours [20]. Moreover, in the present study, like in previous reports, the association between high ferritin levels, outcome variables and some brain injury biomarkers was independent of stroke severity and other prognostic variables. Second, we did not measure molecular markers of oxidative stress, which have not been widely validated in clinical studies [8], so the potential effect of increased iron stores on ROS generation is unknown in patients with acute ischemic stroke. Third, the influence of arterial recanalization on the biological response has not been evaluated, since we did not perform systematic transcranial Doppler ultrasound. Finally, the clinical design of this study does not allow the investigation of the possible synergistic neurotoxic effect of high iron stores and t-PA in acute stroke, which should be investigated in experimental models.

In conclusion, the present findings indicate that brain damage associated with high serum ferritin levels in acute ischemic stroke patients treated with iv. tPA may be mediated by mechanisms linked to excitotoxic damage. The role of inflammation, BBB disruption and oxidative stress in iron-related brain damage needs further research.

\section{Acknowledgements}

Partial results of this investigation were presented at the International Stroke Conference 2007 in San Francisco (CA). This project has been partially supported by grants from the Spanish Ministry of Health (Instituto de Salud Carlos III) FIS PI060788 and RETICSRD06/0026.

\section{References}

[1] T. Aoki, T. Sumii, T. Mori, X. Wang and E.H. Lo, Blood-brain barrier disruption and matrix metalloproteinase- 9 expression during reperfusion injury. Mechanical versus embolic focal ischemia in spontaneously hypertensive rats, Stroke 33 (2002), 2711-2771.

[2] M. Castellanos, N. Puig, T. Carbonell, J. Castillo, J.M. Martínez, R. Rama and A. Dávalos, Iron intake increases infarct volume after permanent middle cerebral artery occlusion in rats, Brain Res 952 (2002), 1-6.

[3] M. Castellanos, R. Leira, J. Serena, M. Blanco, S. Pedraza, J. Castillo and A. Dávalos, Plasma cellular-fibronectin concentration predicts hemorrhagic transformation after thrombolysis therapy in acute ischemic stroke, Stroke 35 (2004), 1671-1676.

[4] M. Castellanos, T. Sobrino, M. Millán, M. García, J. Arenillas, F. Nombela, D. Brea, N. Pérez de la Ossa, J. Serena, J. Vivancos, J. Castillo and A. Dávalos, Serum cellular fibronectin and matrix metalloproteinase-9 predict parenchymal hematoma after thrombolytic therapy in acute ischemic stroke: a multicenter confirmatory study, Stroke 38 (2007), 1855-1859.

[5] M. Castellanos, T. Sobrino, S. Pedraza, O. Moldes, J.M. Pumar, Y. Silva, J. Serena, M. García-Gil, J. Castillo and A. Dávalos, High plasma glutamate concentrations are associated with infarct growth in acute ischemic stroke, Neurology (2008), (in press).

[6] J. Castillo, A. Dávalos, J. Naveiro and M. Noya, Neuroexcitatory amino acids and their relation to infarct size and neurological deficit in ischemic stroke, Stroke 27 (1996), 1060-1065.

[7] J. Castillo, A. Dávalos and M. Noya, Progression of ischemic stroke and excitotoxic aminoacids, Lancet 349 (1997), 79-83.

[8] A. Cherubini, C. Ruggiero, M.C. Polidori and P. Mecocci, Potential markers of oxidative stress in stroke, Free Radical Biology \& Medicine 39 (2005), 841-852.

[9] S. Davis, M.A. Helfaer, R.J. Traystman and P.D. Hum, Parallel antioxidant and antiexcitotoxic therapy improves outcome after incomplete global cerebral ischemia in dogs, Stroke $\mathbf{2 8}$ (1997), 198-205.

[10] A. Davalos, J. Castillo, J. Marrugat, J.M. Fernández-Real, A. Armengou, P. Cacabelos and R. Rama, Body iron stores and early neurological deterioration in acute cerebral infarction, Neurology 54 (2000), 1568-1574.

[11] T. Freret, S. Valable, L. Chazalviel, R. Saulnier, E.T. Mackenzie, E. Petit, M. Bernaudin, M. Boulouard and P. SchumannBard, Delayed administration of deferoxamine reduces brain damage and promotes functional recovery after transient focal cerebral ischemia in the rat, Eur J Neurosci 23 (2006), 1757-1765. 
[12] Y. Gürsoy-Özdemir, A. Can and T. Dalkara, Reperfusioninduced oxidative/nitrative injury to neurovascular unit after focal cerebral ischemia, Stroke 35 (2004), 1449-1453.

[13] G.K. Helal, Systemic administration of $\mathrm{Zn}^{2+}$ during the reperfusion phase of transient cerebral ischaemia protects rat hippocampus against iron-catalysed postischaemic injury, Clin Exp Pharmacol Physiol, [Epub ahead of print], 2008.

[14] O. Hurtado, J. De Cristobal, V. Sanchez, I. Lizasoain, A Cardenas, M.P. Pereira, M.I. Colado, J.C. Leza, P. Lorenzo and M.A. Moro, Inhibition of glutamate release by delaying ATP fall accounts for neuroprotective effects of antioxidants in experimental stroke, FASEB J 17 (2003), 2082-2084.

[15] Kaur J, Zhao Z, Klein GM, Lo EH, Buchan AM, The neurotoxicity of tissue plasminogen activator, J Cereb Blood Flow Metab 24 (2004), 945-963.

[16] H.A. Kontos, Oxygen radicals in cerebral ischemia, Stroke 32 (2001), 2712-2716.

[17] V. Larrue, R.R. von Kummer, A. Muller and E. Bluhmki, Risk factors for severe hemorrhagic transformation in ischemic stroke patients treated with recombinant tissue plasminogen activator: a secondary analysis of the European-Australasian Acute Stroke Study (ECASS II), Stroke 32 (2001), 438-441.

[18] S.H. Mehta, R. Clinton, A. Ergul, A. Tawak and A.M. Dorrance, Neurprotection by tempol in a model of iron-induced oxidative stress in acute ischemic stroke, Am J Physiol 286 (2004), 283-288.

[19] M. Millán, T. Sobrino, M. Castellanos, F. Nombela, J.F. Arenillas, E. Riva, I. Cristobo, M. García, J. Vivancos, J. Serena, M.A. Moro, J. Castillo and A. Dávalos, Increased body iron stores are associated with poor outcome after thrombolytic treatment in acute stroke, Stroke 38 (2007), 90-95.

[20] E. Millerot, A.S. Prigent-Tressier, N.M. Bertrand, P.J.C. Faure, C.M. Mossiat, M.E. Giroud, A.G. Beley and C. Marie, Serum ferritin in stroke: a marker of increased body iron stores or stroke severity? J Cereb Blood Flow Metab 25 (2005), 13861393.

[21] J. Montaner, C.A. Molina, J. Monasterio, S. Abilleira, J.F. Arenillas, M. Ribé, M. Quintana and J́. Alvarez-Sabín, Matrix metalloproteinases-9 pre-treatment level predicts intracranial hemorrhagic complications after thrombolysis in human stroke, Circulation 107 (2003), 598-603

[22] H. Monyer, D.M. Hartley and D.W. Choi, 21-Aminosteroids attenuate excitotoxic neuronal injury in cortical cell cultures, Neuron 5 (1990), 121-126.
[23] M. Ning, K.L. Furie, W.J. Koroshetz, H. Lee, M. Barron, M. Lederer, X. Wang, M. Zhu, A.G. Sorensen, E.H. Lo and P.J. Kelly, Association between t-pa therapy and raised early matrix metalloprotreinase-9 in acute stroke, Neurology 66 (2006), 1550-1555.

[24] A. Patt, I.R. Horesh, E.M. Berger, A.H. Harken and J.E. Repine, Iron depletion or chelation reduces ischemia/ reperfusion-induced edema in gerbil brains, J Pediatr Surg 25 (1990), 224-228.

[25] D.E. Pellegrini-Giampietro, G. Cherici, M. Alesiani, V. Carla and F. Moroni, Excitatory amino acid release and free radical formation may cooperate in the genesis of ischemia-induced neuronal damage, J Neurosci 10 (1990), 1035-1041.

[26] E. Romanos, A.M. Planas, S. Amaro and A. Chamorro, Uric acid reduces brain damage and improves the benefits of rt-pa in a rat model of thromboembolic stroke, J Cereb Blood Flow Metab (2006), 1-7.

[27] M.H. Selim and R.R. Ratan, The role of iron neurotoxicity in ischemic stroke, Ageing Research Reviews 3 (2004), 345-353.

[28] J. Serena, M. Blanco, M. Castellanos, Y. Silva, J. Vivancos, M.A. Moro, R. Leira, I. Lizasoain, J. Castillo and A. Dávalos, The prediction of malignant cerebral infarction by molecular brain barrier disruption markers, Stroke 36 (2005), 1921-1926.

[29] E. Tarkowski, L. Rosengren, C. Blomstrand, C. Wikkelsö, C. Jensen, S. Ekholm and A. Tarkowski, Intrathecal release of pro- and anti-inflammatory cytokines during stroke, Clin Exp Immunol 110 (1997), 492-499.

[30] K. Tsuji, T. Aoki, E. Tejima, K. Arai, S.R. Lee, D.N. Atochin, P.L. Huang, X. Wang, J. Montaner and E. Lo, Tissue plasminogen activator promotes matrix metalloproteinase-9 upregulation after focal cerebral ischemia, Stroke 36 (2005), 1954 1959.

[31] E. Vila, J. Castillo, A. Dávalos and A. Chamorro, Proinflammatory cytokines and early neurological worsening in ischemic stroke, Stroke 31 (2000), 2325-2329.

[32] N. Walhgren, N. Ahmed, A. Dávalos, G.A. Ford, M. Grond, W. Hacke, M.G. Henerici, M. Kaste, S. Kuelkens, V. Larrue, K.R. Less, R.O. Roine, L. Soinne, D. Toni and G. Vanhooren, for the SITS-MOST investigators, Thrombolysis with alteplase for acute ischemic stroke in the Safe Implementation of Thrombolysis in Stroke-Monitoring Study (SITS-MOST): an observational study, The Lancet 369 (2007), 275-282. 


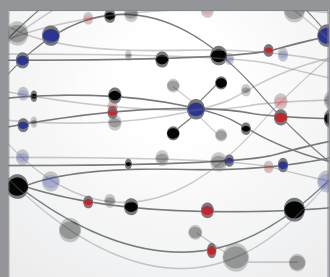

The Scientific World Journal
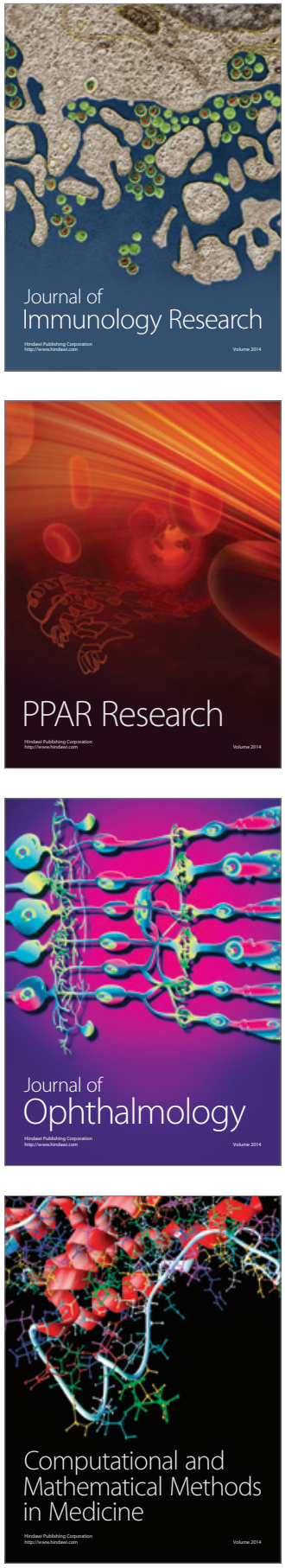

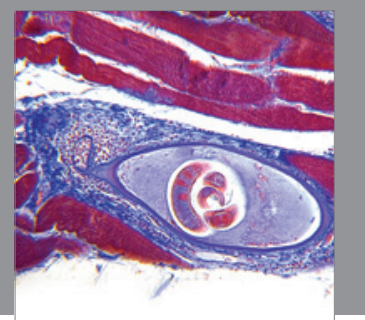

Gastroenterology

Research and Practice
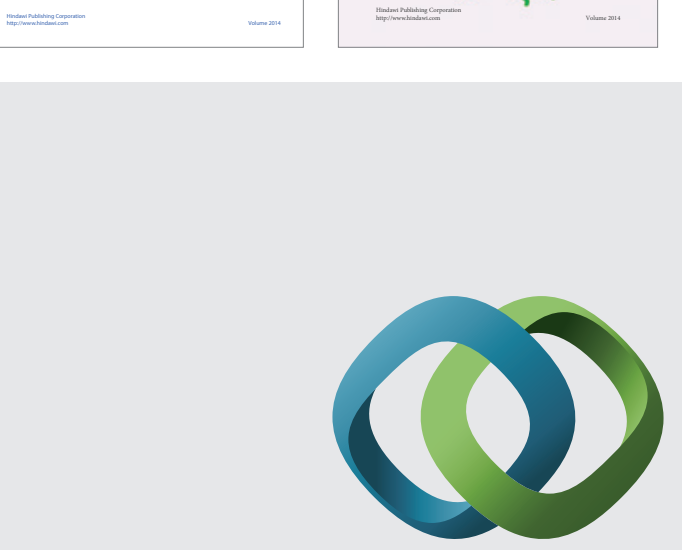

\section{Hindawi}

Submit your manuscripts at

http://www.hindawi.com
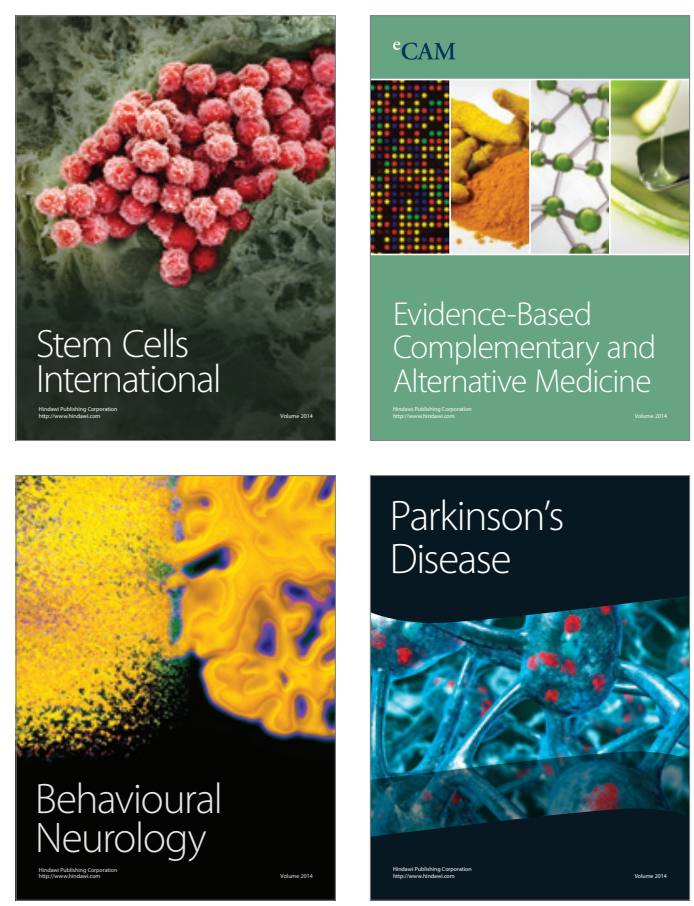

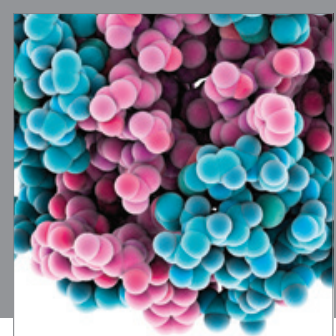

Journal of
Diabetes Research

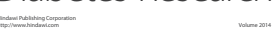

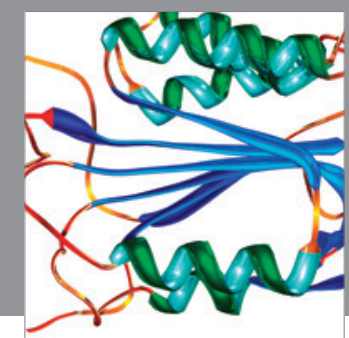

Disease Markers
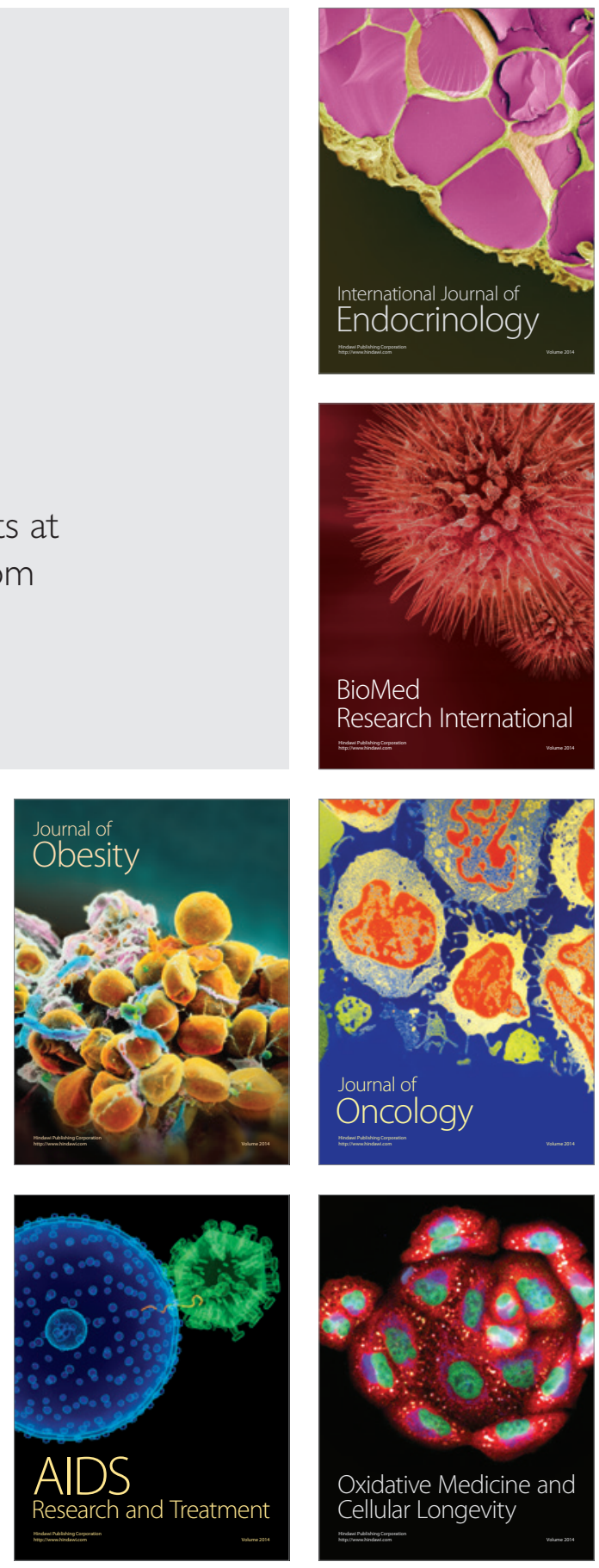